Editorial

\title{
Translating Stem Cell-Based Regenerative Approaches into Clinical Therapies for Musculoskeletal Tissue Repair
}

\author{
Arianna B. Lovati $\mathbb{D}^{1},{ }^{1}$ Bruna Corradetti $\mathbb{D}^{2,3}$ and Roberto Narcisi $\mathbb{D}^{4}$ \\ ${ }^{1}$ Cell and Tissue Engineering Laboratory, IRCCS Istituto Ortopedico Galeazzi, Milan, Italy \\ ${ }^{2}$ Department of Nanomedicine, Houston Methodist Research Institute, Houston, TX, USA \\ ${ }^{3}$ Swansea University Medical School, Swansea, Wales, UK \\ ${ }^{4}$ Department of Orthopaedics and Sports Medicine, Erasmus MC, University Medical Center, Rotterdam, Netherlands
}

Correspondence should be addressed to Arianna B. Lovati; arianna.lovati@grupposandonato.it

Received 22 June 2020; Accepted 22 June 2020; Published 30 January 2021

Copyright (C) 2021 Arianna B. Lovati et al. This is an open access article distributed under the Creative Commons Attribution License, which permits unrestricted use, distribution, and reproduction in any medium, provided the original work is properly cited.

Musculoskeletal tissue repair still represents a challenge in orthopaedics. Stem cell-based regenerative therapies have been widely explored using a variety of approaches, from targeted delivery of stem cells and their derivatives to advanced manufacturing of tissue grafts. Although several studies support the potential of stem cells in musculoskeletal tissue repair, few approaches have been successfully translated into clinics, attributable in part to a lack of well-defined standards of care in preclinical and clinical studies. This translational delay also depends on the intrinsic variability and complexity of the regulatory and legal frameworks worldwide. Stem cell therapy-based strategies vary significantly depending on the nature of the musculoskeletal damage to be restored. While tissue engineering approaches localize stem cells at the site of injury through biomaterials able to create a proregenerative environment, the use of free cells or their derivatives (e.g., secretome, microvesicles, and exosomes) requires limited manipulation and lower processing costs compared to tissue engineering.

This special issue presents the recent advances in the preclinical and clinical use of mesenchymal stem cells (MSCs) or their derivatives (e.g., bone marrow aspirate concentrate, known as BMAC, secretome) for a better understanding of their therapeutic impact.

In this special issue, the manuscript by $\mathrm{C}$. Centeno et al. reports the results of a randomized controlled trial on 25 patients of the treatment of partial to full-thickness supraspinatus tendon tears by BMAC and platelet products as inject- able regenerative therapies. Patients reported a mean $89 \%$ improvement at 24 months, with sustained functional gains, pain reduction, and a size decrease of most tears post-BMAC treatment, suggesting that BMAC injections are a safe and useful alternative to conservative exercise therapy in rotator cuff tears. In their systematic meta-analysis, S. Palombella et al. assessed the current clinical evidence for the efficacy of bone marrow-derived cell-based approaches associated or not with bone scaffolds to treat bone nonunions. Specifically, in the review, the authors indicate that BMAC or $\mathrm{BMAC} /$ scaffold is the primary choice versus bone marrowderived stromal cells (BMSCs) to treat nonunions with a successful healing at a midterm follow-up (6-12 months). For the clinical translation of cell-based products, rigid protocols need to be established. In their work, R. B. Dias et al. described the manufacture of GMP-grade BMSC in compliance with Brazilian regulatory guidelines for cells intended for use in humans. Indeed, in vitro-expanded BMSCs have long been proposed for the treatment of complex bone injuries due to their inherent potential to differentiate into multiple skeletal cell types, modulate inflammatory responses, and support angiogenesis.

In the field of musculoskeletal tissue repair, nerve injuries represent a prominent clinical issue. Damaging the connection between muscle and neural fibers, for example, leads to muscle atrophy, with also a known negative impact on the differentiation capacity of the resident tissue-specific progenitor cells. In their review, Y. Wang et al. focused on the mechanism of 
action, the role and the possible regenerative medicine applications of Adipose Stem Cells (ASCs) for diseases affecting the nervous system, overall indicating how these cells can become one of the most important cell-based tools for the treatment of neural pathologies in the future.

This special issue also introduces the reader to basic research aiming at identifying an alternative source of stem cells with improved differentiative potential into musculoskeletal tissues. The study conducted by H. E. Owston et al. assesses the migration and differentiation properties of multipotent stromal cells from clinically accessible human periosteum compared to BMSCs, demonstrating that periosteum represents a consistently rich source of highly proliferative multipotent stromal cells compared to donor-matched BMSCs with similar osteochondral capacity and lower adipogenic potential. In the same line, in their in vivo study, E. R. Moore et al. highlighted the importance of periosteal Prx1expressing cells in contributing to the mechanically induced bone formation. Interestingly, the authors were able to show how primary cilia are necessary for a complete mechanical response of the Prx1-expressing cells, contributing to increase the knowledge around the role of this, still poorly understood, cellular organelle during bone (re)generation.

The relevance of the immunomodulatory potential of stem cells in the treatment of musculoskeletal diseases has been investigated by $\mathrm{M}$. Viganò et al. The authors proposed the microfragmented adipose tissue $(\mu \mathrm{FAT})$ as a convenient autologous product for the application of MSC-based therapies in clinical settings. In particular, they demonstrated its efficacy to favor tissue healing in the context of tendon inflammation, highlighting its capability to counteract the catabolic and fibrotic response elicited by the proinflammatory interleukin-1 beta (IL-1b) on tendon cells and providing insights on the mechanism of action of this therapeutic approach for the treatment of tendon disorders.

The special issue widely reports preclinical studies testing the efficacy of stem cell-based therapies in animal models and their relevance with respect to the human pathology in orthopaedics. In their study, M. Bottagisio et al. demonstrated the beneficial role of undifferentiated BMSCs loaded within decellularized xenografts undergoing a stretchperfusion culture as an immunomodulatory weapon reducing the inflammatory process in tendon ruptures. This study associates the advantages of in vitro expanded and dynamically cultured BMSCs with new biologically relevant biomaterials with the potential to promote tendon reconstruction. In particular, J. Zhang et al. address an important clinical problem for the restoration of injured musculoskeletal tissues, which is related to the poor intrinsic healing potential of tendon and ligament tissue. The authors investigated the influence of Fibroblast Growth Factor-2- (FGF-2-) transfected human amniotic MSCs (hAMSCs) seeded on a human acellular amniotic membrane scaffold on tendon-to-bone healing process, using in vitro tests and a rabbit in vivo model. Their data demonstrate a significantly better healing of a tendon-to-bone junction, compared to the previously used biological materials.

Animal models are also a useful tool to investigate the cell fate/engraftment by tracking imaging in orthopaedics. The original article by C. Horstmeier et al. investigated in vivo the magic angle imaging technique to track ASCs labelled with superparamagnetic iron oxide particles (SPIO) and to discriminate SPIO-induced artefacts from the surrounding tendon tissue in equine digital flexor tendons.

Overall, in this research topic, we wanted to underline the importance of verifying the safety and efficacy of stem cellbased approaches, as well as the development of relevant preclinical studies, both in vitro and in vivo. Moreover, a multidisciplinary approach is required to better elucidate the mechanisms of stem cell differentiation, function, and tissue healing. Even more importantly, a good design of clinical trials should be implemented for the final validation of stem cell-based therapies in orthopaedics.

\section{Conflicts of Interest}

The editors declare that they have no conflicts of interest regarding the publication of this special issue.

\section{Acknowledgments}

The editors thank all the authors and reviewers who contributed to the research topic and success of this special issue. We would like to thank the editorial board members of Stem Cells International for promoting this issue and for the assistance and help during the reviewing process. R.N. is financially supported by a TTW Perspectief Grant: William Hunter Revisited from the Dutch National Organization NWO (P15-23) and by the INtegrate grant from the AOFoundation Davos in Switzerland (ARI CRP OCD-Integrate). B.C. acknowledges support through the Sêr Cymru II programme, funded by the European Commission through the Horizon 2020 research and innovation programme under the Marie Skłodowska-Curie Actions (MSCA) COFUND scheme and the Welsh European Funding Office (WEFO) under the European Regional Development Fund (ERDF) (grant agreement 663830).
Arianna B. Lovati Bruna Corradetti Roberto Narcisi 